\title{
Learning from Data Streams using Kernel Least-Mean- Square with Multiple Kernel-Sizes and Adaptive Step-Size
}

DOI:

10.1016/j.neucom.2019.01.055

\section{Document Version}

Accepted author manuscript

Link to publication record in Manchester Research Explorer

\section{Citation for published version (APA):}

Garcia-vega, S., Zeng, X., \& Keane, J. (2019). Learning from Data Streams using Kernel Least-Mean-Square with Multiple Kernel-Sizes and Adaptive Step-Size. Neurocomputing. https://doi.org/10.1016/j.neucom.2019.01.055

\section{Published in:}

Neurocomputing

\section{Citing this paper}

Please note that where the full-text provided on Manchester Research Explorer is the Author Accepted Manuscript or Proof version this may differ from the final Published version. If citing, it is advised that you check and use the publisher's definitive version.

\section{General rights}

Copyright and moral rights for the publications made accessible in the Research Explorer are retained by the authors and/or other copyright owners and it is a condition of accessing publications that users recognise and abide by the legal requirements associated with these rights.

\section{Takedown policy}

If you believe that this document breaches copyright please refer to the University of Manchester's Takedown Procedures [http://man.ac.uk/04Y6Bo] or contact uml.scholarlycommunications@manchester.ac.uk providing relevant details, so we can investigate your claim.

\section{OPEN ACCESS}




\title{
Learning from Data Streams using Kernel Least-Mean-Square with Multiple Kernel-Sizes and Adaptive Step-Size
}

\author{
Sergio Garcia-Vega ${ }^{\mathrm{a}, *}$, Xiao-Jun Zeng ${ }^{\mathrm{a}}$, John Keane ${ }^{\mathrm{a}}$ \\ ${ }^{a}$ School of Computer Science, University of Manchester, Kilburn Building, Oxford Road, \\ Manchester, M13 9PL, United Kingdom
}

\begin{abstract}
A learning task is sequential if its data samples become available over time; kernel adaptive filters (KAFs) are sequential learning algorithms. There are three main challenges in KAFs: (1) selection of an appropriate Mercer kernel; (2) the lack of an effective method to determine kernel-sizes in an online learning context; (3) how to tune the step-size parameter. This work introduces a framework for online prediction that addresses the latter two of these open challenges. The kernel-sizes, unlike traditional KAF formulations, are both created and updated in an online sequential way. Further, to improve convergence time, we propose an adaptive step-size strategy that minimizes the mean-square-error (MSE) using a stochastic gradient algorithm. The proposed framework has been tested on three real-world data sets; results show both faster convergence to relatively low values of MSE and better accuracy when compared with KAF-based methods, long short-term memory, and recurrent neural networks.
\end{abstract}

Keywords: Learning from data streams; Sequence prediction; Kernel least-mean-square; Kernel-size; Step-size.

\footnotetext{
${ }^{*}$ Corresponding author

Email addresses: sergio.garcia-vega@manchester.ac.uk (Sergio Garcia-Vega),

x.zeng@manchester.ac.uk (Xiao-Jun Zeng), john.keane@manchester.ac.uk (John Keane)
} 


\section{Introduction}

A learning task is sequential if its training samples become available over time, usually one sample at a time. The sequence imposes an order on the samples; this order must be preserved when training models and making predictions [1. The goal being to extract knowledge from a continuous sequence of data records, e.g., financial markets, network traffic, weather conditions, among others [2, 3, 4].

Sequence prediction models, unlike traditional classification and regression problems, have memory and can learn temporal dependence between observations [5. For example, long short-term memory (LSTM) is a neural network $(\mathrm{NN})$ that can pass information across time and model long-term dependencies using gating mechanisms [6, 7]. Although $\mathrm{NN}$ approaches have proven useful in a wide variety of real-world problems [8, 9, 10, 11, their application in sequence prediction tasks is subject to: (1) the assumption that the test data set has similar samples to the training data [12]; (2) the need to retrain the model at regular intervals, which requires both significant computing and storage resources [13].

Kernel Adaptive Filters (KAFs), like NNs, are nonlinear approximators. These algorithms combine the universal approximation property of NNs and the convex optimization of linear adaptive filters. In contrast to NNs, KAFs are non-parametric, have moderate computational complexity and convex optimization [14. This is an advantage with respect to NNs, where training is always problematic because the system state can get stuck in local minima [15]. The learning scheme of KAFs, unlike NNs, is a combination of error-correction and memory-based learning, which means that they do not require the whole training set to start learning the model. In contrast, as the samples arrive, predictions are obtained while the model is updated sequentially at the same time, which is useful in real-time applications. In other words, unlike NNs, the model is learned using a single pass through the entire training set in KAF algorithms. As a result, KAFs have been shown to be an efficient alternative to identify non-linear systems in online sequential learning [16, 17, 18. 
Algorithms based on KAFs operate in a very special Hilbert space of functions called a reproducing kernel Hilbert space (RKHS). Operating with such functions become much easier in RKHS if the computation is restricted to inner products, which is the idea behind kernel methods. A variety of KAF algorithms have been proposed such as kernel least-mean-square (KLMS) [19] and kernel affine projection [20] - others include [21, 22]. However, there remain three main challenges in KAF algorithms: selection of an appropriate Mercer kernel, determination of kernel parameter and tuning of the step-size [23]. Here the Mercer kernel is assumed to be the Gaussian kernel due to its universal approximating capability, desirable smoothness and numeric stability [14].

In this work, we address two of these open challenges;

1. For the Gaussian kernel, the kernel parameter, also known as kernel-size, is used to define similarity between data points - if the kernel-size is too large, all data will look similar; in contrast, if the kernel-size is too small, all data will look distinct. The kernel-size is usually calculated using crossvalidation [24, penalizing functions 25], plug-in methods [26], Silverman's rule [27], or manual selection. However, such methods are unsuitable for determining an optimal kernel-size in KAFs, as they are either batch mode methods or originate from a different problem [23].

Multiple-kernel-learning methods have been proposed to address the problem of kernel-size in KAFs. For example, mixture KLMS employs a Gaussian kernel with multiple kernel-sizes [28]. However, a pool of predefined kernel-sizes is needed in advance, meaning that the algorithm cannot create new kernel-sizes in an online way. Hence, the algorithm may be unable to adjust to abrupt changes in the system. Despite this, the idea of switching the models at different instances of time may be useful to identify non-stationary systems. A method to find the kernel-size of a KAF algorithm in an online sequential way was proposd in [23]. Results show that the kernel-size can converge to an appropriate value; consequently, there is an improvement in both convergence and accuracy of the KAF algo- 
rithm. However, when the environment changes back to a previous mode, the algorithm has no inherent mechanism for recall and has to relearn the structure from scratch, i.e., as the kernel-size is updated sequentially, the method throws away previously learned kernel-sizes, but the appropriate kernel-size for the current sample — viewed from multiple-kernel-learning — may be one learned previously.

2. The step-size is the compromise between convergence time and misadjustment, i.e., increasing step-size reduces convergence time but increases misadjustment. Risk of overfitting increases if the step-size is too large, while a smaller step-size helps generalization [14. Although many KAF methods use this parameter to compute predictions, they do not optimize step-size within the context of adaptive filtering with kernels [19, 21, 29, 30]; for example, recent work has proposed kernel-size optimization [23], however, that work does not optimize the step-size parameter (though it is noted as "future work"). In practice, this parameter is calculated offline, hence it remains unchanged through iterations. Consequently, online adaptation of KAF algorithms is highly conditional on the choice of step-size.

We introduce a framework for online prediction based on KAFs. This framework learns from a continuous sequence of data records and rapidly adapts to changes in the system. Unlike traditional KAF formulations, our approach does not need a predefined set of kernel-sizes; rather, here, the kernel-sizes are created in an online sequential way. Further, we propose an adaptive step-size to improve convergence time.

The framework is validated on three real-world data sets: (1) internet traffic data from a private internet service provider with centres in eleven European cities? (2) the mid-prices of two major currencies in the foreign exchange market? (3) Tesla stock prices from its initial public offering? Simulation results

\footnotetext{
${ }^{1}$ The dataset is publicly available at http://www3.dsi.uminho.pt/pcortez/series/A5M.txt

${ }^{2}$ The dataset is publicly available at https://www.dukascopy.com

${ }^{3}$ The dataset is publicly available at https://www.kaggle.com/rpaguirre/tesla-stock-price
} 
show that the framework is robust to noisy and non-stationary conditions. In comparison to similar methods, our proposal converges more quickly and achieve better accuracy. Overall, relatively lower values of mean squared error are obtained in all tested data sets. NN methods such as LSTM give competitive performance; however, the LSTM model must be retrained regularly in sequence prediction tasks. The proposed framework, unlike LSTM, does not need the whole training set to start learning the model. In contrast, as samples arrive, predictions are generated while the model is updated sequentially at the same time, which is useful in real-time applications.

In summary, the contributions of this paper are: (1) an online-sequential learning algorithm to address the kernel-size problem in KAFs; (2) an adaptive step-size in KAFs; and (3) a framework for online prediction based on KAFs.

The remainder of the paper is structured as follows: KAF algorithms are briefly described in Section 2, the proposed framework is introduced in Section 3 . Section 4 gives the experiment settings of this work; and Sections 5 and 6 provides simulation results and conclusions, respectively.

\section{Technical Background}

A learning algorithm is sequential if, for any given training examples $\left\{\boldsymbol{u}_{1}, y_{1}\right\}$, $\left\{\boldsymbol{u}_{2}, y_{2}\right\}, \ldots,\left\{\boldsymbol{u}_{t}, y_{t}\right\}$, it produces a sequence of hypotheses $h_{1}, h_{2}, \ldots, h_{t}$ such that $h_{t}$ depends not only on the previous hypothesis $h_{t-1}$, but also on the current sample $\left\{\boldsymbol{u}_{t}, y_{t}\right\}$ [14], e.g., linear adaptive filters. These filters adjust their free parameters automatically in response to statistical variations in the environment. In this section, we briefly describe the technical background used throughout the paper. First, we describe sequential learning in linear models (Section 2.1). Then, we introduce the simplest and most commonly used form of a kernel-based adaptive filter (Section 2.2).

\subsection{Least-Mean-Square Algorithm}

The simplest and most commonly used form of an adaptive filtering algorithm is least-mean-square (LMS). Suppose the goal is to learn a continuous 
input-output mapping $f: \mathbb{U} \rightarrow \mathbb{R}$ based on a sequence of input-output examples $\mathcal{T}=\left\{\boldsymbol{u}_{t}, y_{t}: t \in[1, N]\right\}$, where $\mathbb{U} \subset \mathbb{R}^{M}$ is the input domain, $\boldsymbol{u}_{t} \in \mathbb{R}^{M}$ is an input vector, and $y_{t} \in \mathbb{R}$ is the desired output. The LMS algorithm operates by minimizing the instantaneous cost function $J_{t}=\frac{1}{2} e_{t}^{2}$, where the prediction error is $e_{t}=y_{t}-\mathbf{w}_{t-1}^{T} \boldsymbol{u}_{t}$. Thus, the LMS algorithm assumes a linear model and uses the following procedure:

$$
\left\{\begin{aligned}
\mathbf{w}_{0} & =0 \\
e_{t} & =y_{t}-\mathbf{w}_{t-1}^{T} \boldsymbol{u}_{t} \\
\mathbf{w}_{t} & =\mathbf{w}_{t-1}+\eta e_{t} \boldsymbol{u}_{t}
\end{aligned}\right.
$$

where $\eta$ is the step-size parameter and $\mathbf{w}_{t}$ is the estimate of the optimal weight at iteration $t$. However, if the mapping between $y$ and $\boldsymbol{u}$ is highly nonlinear, then LMS is likely to perform poorly.

\subsection{Kernel Adaptive Filters (KAFs)}

KAFs naturally create a growing radial-basis function network (RBFN), that learns the network topology and, at the same time, adapts the free parameters directly from the data. KAF methods reproduce RKHS, using a linear adaptive structure to obtain nonlinear filters in the input space. RKHS plays a central role in providing linearity, convexity and universal approximation capability [14].

To overcome the limitation of linearity in LMS, KAF employs a kernelinduced mapping $\varphi: \mathbb{U} \rightarrow \mathbb{F}$ to transform the input $\boldsymbol{u}_{t}$ into a high-dimensional feature space $\mathbb{F}$, which is an inner product space, as $\varphi\left(\boldsymbol{u}_{t}\right)$. Any Mercer kernel $\kappa\left(\boldsymbol{u}_{t}, \boldsymbol{u}^{\prime}\right)$, where $\kappa: \mathbb{U} \times \mathbb{U} \rightarrow \mathbb{R}^{+}$and $\boldsymbol{u}^{\prime}$ is a new input, induces a mapping $\varphi$ such that the following relationship, termed the kernel trick [31, holds:

$$
\varphi\left(\boldsymbol{u}_{t}\right)^{\top} \varphi\left(\boldsymbol{u}^{\prime}\right)=\kappa\left(\boldsymbol{u}_{t}, \boldsymbol{u}^{\prime}\right)
$$

The KLMS algorithm, the simplest in the KAF family, is a sequential estimator of $f$ (see Section 2.1) such that $f_{t}$ is updated using the last estimate $f_{t-1}$ and the current example $\left\{\boldsymbol{u}_{t}, y_{t}\right\}$, yielding the following sequential rule in the 
original input space [19]:

$$
\left\{\begin{array}{l}
f_{0}=0 \\
e_{t}=y_{t}-f_{t-1}\left(\boldsymbol{u}_{t}\right) \\
f_{t}=f_{t-1}+\eta e_{t} \kappa_{\sigma}\left(\boldsymbol{u}_{t}, \cdot\right)
\end{array}\right.
$$

where $e_{t} \in \mathbb{R}$ is the prediction error, $f_{t}$ denotes the learned mapping at iteration

$t, \eta \in \mathbb{R}^{+}$is the step-size parameter, and $\kappa_{\sigma}(\cdot, \cdot) \in \mathbb{R}^{+}$is a Mercer kernel with a kernel-size $\sigma \in \mathbb{R}^{+}$that controls the mapping smoothness [31. This sequential rule produces a growing RBFN by allocating a new kernel unit for every new example with $\boldsymbol{u}_{t}$ as the center and $\eta e_{t}$ as its coefficient, which poses time-space complexity issues for continuous adaptation scenarios. Note, the set of centers and coefficients are also known as dictionary and weights, respectively.

\section{A framework for online prediction}

We develop a framework for online prediction that addresses two main challenges of $\mathrm{KAF}$ algorithms:

1. an algorithm that uses multiple kernel-sizes in online sequential learning to address the kernel-size problem (Section 3.1);

2. an online technique to optimize the step-size (Section 3.2. .

\subsection{Multiple kernel-sizes in online sequential learning}

We propose an online algorithm to address the kernel-size problem in the KLMS algorithm. Our proposal, unlike traditional multiple-kernel-learning formulations, does not need a predefined set of kernel-sizes. Rather, here the algorithm creates a pool of kernel-sizes in an online sequential way. In addition, the appropriate kernel-size is selected for every new input sample. This allows the algorithm to adjust to abrupt changes in the system, which is useful in 
non-stationary conditions. More formally, the kernel-size can be computed a: 4

$$
\sigma_{t}=\sigma_{t-1}+\frac{\rho e_{t-1} e_{t}\left\|\boldsymbol{u}_{t-1}-\boldsymbol{u}_{t}\right\|^{2} \kappa_{\sigma_{t-1}}\left(\boldsymbol{u}_{t-1}, \boldsymbol{u}_{t}\right)}{\sigma_{t-1}^{3}},
$$

from Equation (4), the following observations can be made [23]: (1) the gradient direction depends on the signs of the prediction errors $e_{t-1}$ and $e_{t}$. If there is little sign change, the desired mapping is likely a "smoothing function"; in contrast, if the sign changes frequently, the desired mapping is likely a "zig zag function"; (2) the magnitude of the gradient depends on $\sigma_{t-1}$ through $\kappa_{\sigma_{t-1}}\left(\boldsymbol{u}_{t-1}, \boldsymbol{u}_{t}\right) / \sigma_{t-1}^{3}$. For the case $\boldsymbol{u}_{t} \neq \boldsymbol{u}_{t-1}$, this term will approach zero when $\sigma_{t-1}$ is very small or very large. Although gradient goes to zero, it does not imply that $\sigma_{t}$ is bounded, however with a proper initial value, the kernel-size will be adjusted within a reasonable range; (3) if the initial kernel-size $\sigma_{1}$ is inappropriately chosen, initial convergence speed can be very slow. In this case, the suitable initial kernel-size can be selected using a method such as Silverman's rule [27.

Equation (4) allows the kernel-size to be computed in an online sequential way. However, when the environment changes back to a previous mode, the online technique to optimize the kernel-size has no inherent mechanism for recall and thus must relearn the structure from scratch. In other words, as the kernelsize is updated sequentially, the prediction $f_{t}$ (see Equation (3p) for the input sample $\boldsymbol{u}_{t}$ is calculated using $\sigma_{t}$, but the appropriate kernel-size for $\boldsymbol{u}_{t}$ - viewed from multiple-kernel-learning - may have been learned previously, i.e., $\sigma_{1}, \ldots$, $\sigma_{t-2}, \sigma_{t-1}$.

Rather than throw away previously learned kernel-sizes, we use them to build a pool of kernel-sizes in an online sequential way. Then, for each new input sam-

\footnotetext{
${ }^{4}$ Where: (1) the kernel-size at iteration $t-1$ is $\sigma_{t-1}$; (2) the free parameter for the kernelsize adaptation is $\rho$; (3) the prediction errors at time $t-1$ and $t$ are $e_{t-1}$ and $e_{t}$, respectively; (4) the input samples at time $t-1$ and $t$ are $\boldsymbol{u}_{t-1}$ and $\boldsymbol{u}_{t}$, respectively; (5) $\|\cdot\|$ denotes the $\ell_{2}$ norm; (6) $\kappa_{\sigma}(\cdot, \cdot)$ is a Mercer kernel. In our proposal, the Mercer kernel is assumed to be the Gaussian kernel, i.e., $\kappa_{\sigma}\left(\boldsymbol{u}_{t-1}, \boldsymbol{u}_{t}\right)=\exp \left(-\left\|\boldsymbol{u}_{t-1}-\boldsymbol{u}_{t}\right\|^{2} / 2 \sigma^{2}\right)$.
} 
ple, the appropriate kernel-size is selected from this pool. In practice, once the algorithm selects the appropriate kernel-size for the current sample $\boldsymbol{u}_{t}$, the next step is to apply a KLMS-based algorithm to perform the prediction task. Note that the KLMS-based algorithm provides a dictionary 5 and its weights, which are used in a linear combination to obtain the predictions (see Equation (3)).

We provide a step-by-step description of how we create the kernel-sizes in an online sequential way (Lines refer to Algorithm 1):

- Start with an initial kernel-size $\sigma_{1}$, which is provided in advance (Line 1).

- The centroid of the initial kernel-size $c_{1} \in \mathbb{R}^{M}$ is created using the first input sample $\boldsymbol{u}_{1}$ (Line 4).

- The first input sample is stored in the dictionary of the initial kernel-size $\mathcal{C}_{1}$ (Line 5).

- The new sample $\boldsymbol{u}_{t}$ and the centroids are projected into a high-dimensional feature space via a reproducing kernel (Line 12). This is also known as coherence (a fundamental parameter that characterizes a dictionary in linear sparse approximation problems) within the context of adaptive filtering with kernels [33]. The coherence criterion suggests inserting the candidate input $\boldsymbol{u}_{t}$ as a new center if its coherence $\max _{1 \leq i \leq|\mathbf{K}|} \kappa_{\sigma_{i}}\left(\boldsymbol{u}_{t}, c_{i}\right)$ remains below a given threshold $\delta \in[0,1]$. The main rationale behind the previous strategy is to provide both the level of sparsity and the coherence of the kernel-sizes created during learning 34 .

- The centroid threshold $\delta$ determines whether or not a new kernel-size should be created:

1. $\max _{1 \leq i \leq|\mathbf{K}|} \kappa_{\sigma_{i}}\left(\boldsymbol{u}_{t}, c_{i}\right) \geq \delta$ : This means that one of the current kernelsizes is appropriate for the new sample; thus, there is no need to create a new kernel-size and the most similar kernel-size $i^{*}$ is selected

\footnotetext{
${ }^{5}$ A selected set of input samples used to estimate the nonlinear model [32].
} 
for the new sample $\boldsymbol{u}_{t}$ (Line 11). Next, the centroid of the most similar kernel-size $c_{i^{*}}$ is updated (Lines 13 14).

2. $\max _{1 \leq i \leq|\mathbf{K}|} \kappa_{\sigma_{i}}\left(\boldsymbol{u}_{t}, c_{i}\right)<\delta$ : A new kernel-size is created (Line 17). Then, the current sample $\boldsymbol{u}_{t}$ is used to create the centroid of the new kernelsize $c_{|\mathbf{K}|+1}$ (Lines 18 19. After that, the dictionary and the pool of kernel-sizes are updated (Lines 21 26).

- Following selection of the appropriate kernel-size for the current sample, the next step applies a KLMS-based algorithm to perform the prediction task (Line 29p. Lastly, the kernel-size and the step-size are updated before the next sample $\boldsymbol{u}_{t+1}$ arrives (Lines 32 36).

The general scheme of the proposed framework for online prediction at iteration $t$ is summarized in Figure 1 .

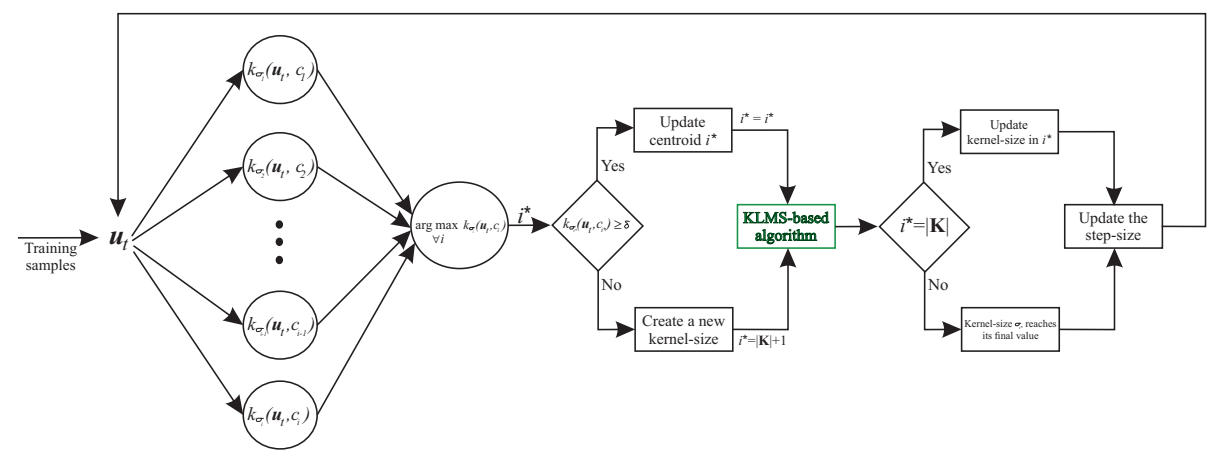

Figure 1: Proposed framework for online prediction at iteration $t$.

Remark 1 (Centroid). In Algorithm 1, unlike the original formulation [35], we use the centroids to determine the appropriate kernel-size for each new input sample $\boldsymbol{u}_{t}$. The new input sample is projected in a high-dimensional feature space. This is done using the centroids $c_{i}$ and their kernel-sizes $\sigma_{i}$, i.e., in this paper, the centroid is a single representative of each kernel-size.

Remark 2 (Kernel-size optimization). Optimization of the kernel-size is performed only on the most recently created kernel-size (Line 33), which means 
that all other kernel-sizes have a fixed value (Line 35). Once a new kernel-size is created $|\boldsymbol{K}|+1$, optimization of the kernel-size $|\boldsymbol{K}|$ stops (Line 16 ). Consequently, the pool of kernel-sizes $\boldsymbol{K}$ is created and updated in an online sequential way.

Remark 3 (KLMS-based algorithm). It is possible to use any algorithm based on KLMS (Line 29).

Remark 4 (Knowledge-transfer). When a new $\sigma$ is added to the pool of kernelsizes, its dictionary and weights start with the dictionary of the most similar kernel-size $i^{*}$ at time $t$ (Lines 21.24); thus, it is possible to have overlaps in the dictionaries of the kernel-sizes. This strategy can be viewed as a smoothing procedure, which is also known as knowledge-transfer; in the worse case, the dictionary of the new $\sigma$ will retain a dictionary size equivalent to KLMS. Note, if we allow the dictionary of the new $\sigma$ to be initialized from scratch, as in traditional adaptive filtering, this will result in a discontinuity of performance in time. In practice, the kernel-sizes are automatically created in the early stages of training, meaning that overlaps are small. Our proposal uses previously learned knowledge with the appropriate kernel-size to enhance prediction. The knowledge-transfer scheme has similarities to a previously proposed method [35]; the key difference in the two strategies is that our proposal has copied not only the weights but also the dictionary of the most similar kernelsize. This better utilizes the kernel-sizes, avoids large discontinuities in learning time, provides more efficient training in non-stationary conditions, and in some circumstances may yield a more compact network [21].

Remark 5 (Computational and memory issues). In our proposal, the appropriate kernel-size is selected when a new sample arrives. Then, the prediction task is performed using this kernel-size together with its dictionary and weights. Therefore, as mentioned in Remark 4, the selected dictionary will retain a dictionary size equivalent to KLMS in the worst case (see Figure 2). 


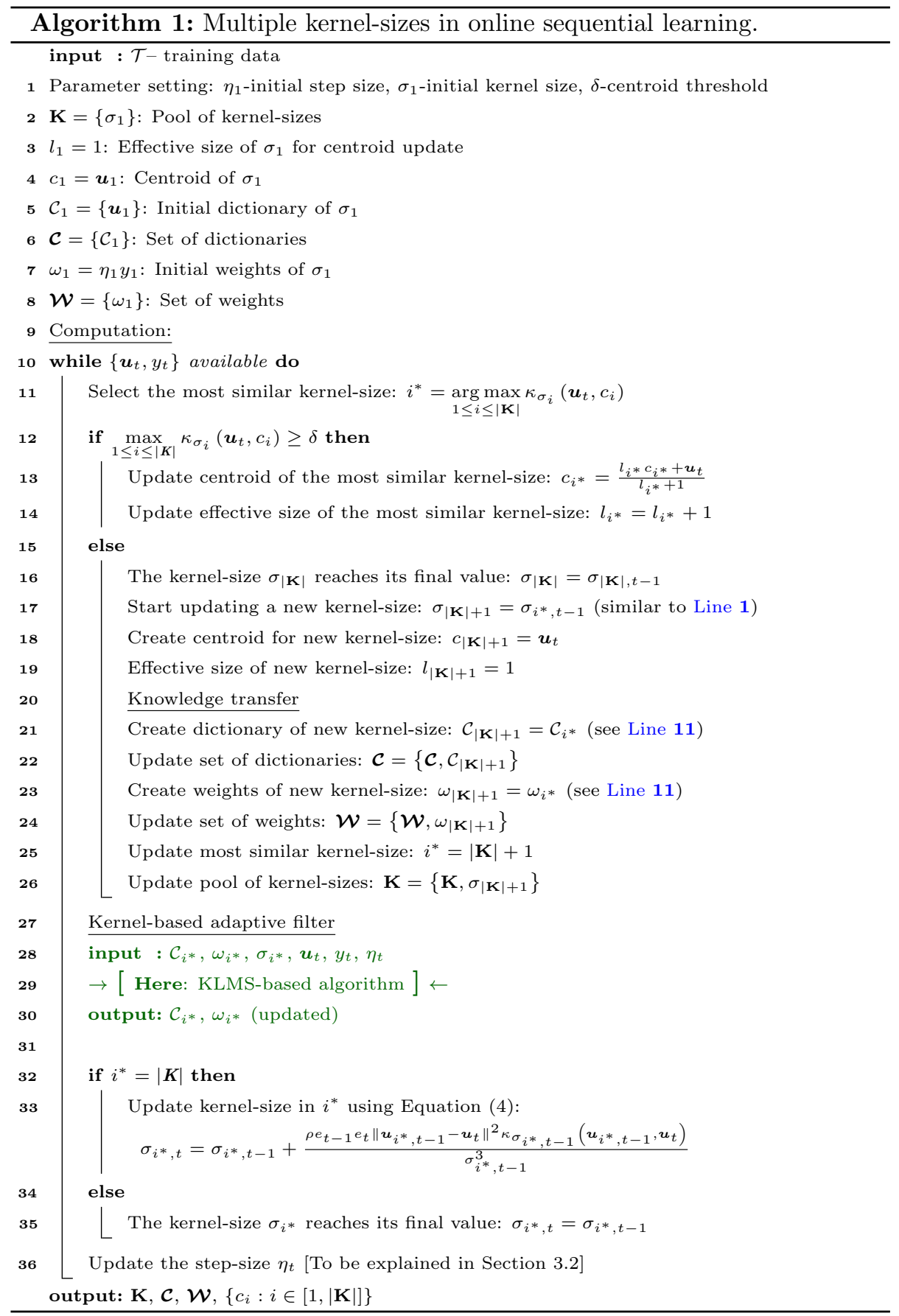


To clarify how Alg. 1 works in practice, assume that the input samples are $\boldsymbol{u}_{t} \in \mathbb{R}^{3}$. The first step is to start a kernel-size $\oint^{6}$ with its dictionary (see Figure 2(a) . At that moment, the update procedure (see Equation (4)) on the first kernel-size creates a new kernel-size. Then, at time $t=4$, the similarity between $\boldsymbol{u}_{4}$ and the centroid of the first kernel-size $c_{1}$ is calculated. However, $\kappa_{\sigma_{1, t=3}}\left(\boldsymbol{u}_{4}, c_{1}\right)$ is not greater than $\delta$, indicating the presence of a new dynamic in the system. Consequently, a new kernel-size is created and $\boldsymbol{u}_{4}$ is its initial centroid 7 (see Figure 2(b) . Finally, at time $t=7$, a new kernel-size is again created and the process is repeated in a similar way as at time $t=4$ (see Figure 2(c) . Note that, at time $t=24$ : (1) kernel-size optimization is performed only on the last created kernel-size; (2) the first and second kernel-size already have a fixed value, i.e., $\sigma_{1, t=3}$ and $\sigma_{2, t=6}$, respectively (see Figure 2(d)).

Finally, a major advantage of Alg. 1 1 is that the prediction is performed based on a relatively low number of samples. This is because for every new sample we select its best possible kernel-size as well as its closest dictionary (from the point of view of data distribution). For example, when $\boldsymbol{u}_{24}$ arrives, the prediction task is performed using only the $\mathbf{5}$ samples of cluster 3 in the dictionary of the third kernel-size, i.e., $\boldsymbol{u}_{3}, \boldsymbol{u}_{6}, \boldsymbol{u}_{8}, \boldsymbol{u}_{16}$, and $\boldsymbol{u}_{24}$ (see Figure 2(d)]. In comparison to the traditional KLMS algorithm, the same prediction is calculated using $\mathbf{2 4}$ samples, i.e., $\boldsymbol{u}_{1}, \boldsymbol{u}_{2}, \boldsymbol{u}_{3}, \cdots, \boldsymbol{u}_{24}$. For this reason, Alg. 1 runs much faster than KLMS and variants.

\footnotetext{
${ }^{6}$ The syntax $\sigma_{1, t=1}$ stands for the first kernel-size at time $t=1$.

${ }^{7}$ Note, when the second kernel-size is created at time $t=4$ : (1) optimization of the first kernel-size stops, and, from this point on, its kernel-size will be $\sigma_{1, t=3} ;(2)$ the second kernelsize starts with the last known $\sigma$, i.e., the first kernel-size at time $t=3$; (3) optimization on the second kernel-size stops when a new kernel-size is again created; (4) to avoid discontinuities in learning, the samples in the dictionary of the first kernel-size - $\boldsymbol{u}_{1}, \boldsymbol{u}_{2}$, and $\boldsymbol{u}_{3}$ - are copied to the dictionary of the new kernel-size (knowledge transfer); (5) the three clusters inside the dictionaries of the kernel-sizes are generated by the KLMS-based algorithm [35].
} 


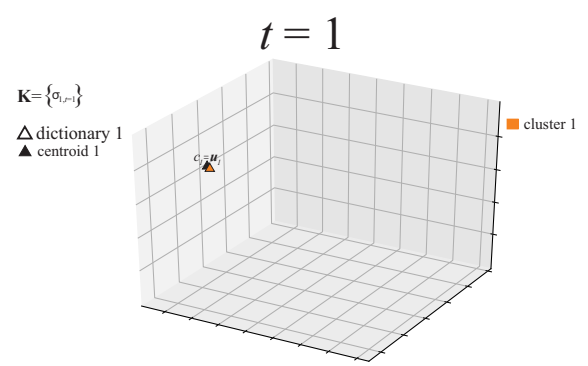

(a) Initialization.

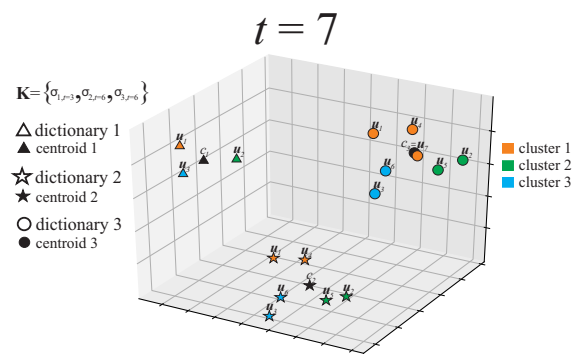

(c) Sample $\boldsymbol{u}_{7}$ arrives.

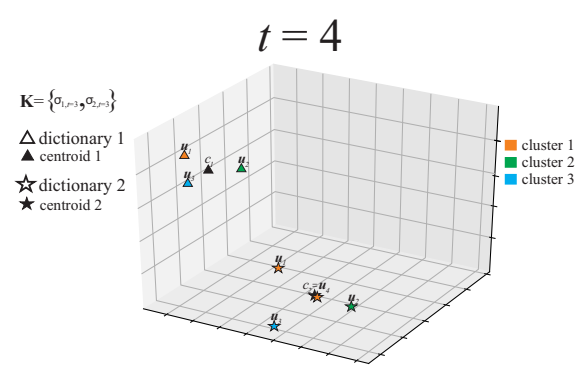

(b) Sample $\boldsymbol{u}_{4}$ arrives.

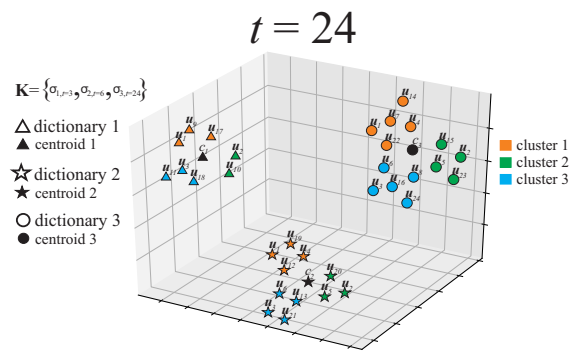

(d) Sample $\boldsymbol{u}_{24}$ arrives.

Figure 2: Multiple kernel-sizes in online sequential learning.

\subsection{An online technique to optimize step-size}

In this section, we propose an adaptive step-size strategy, another well-known challenge in KAFs.

At each iteration, the step-size of the KLMS is optimized using the previous step-size and the current prediction error. More formally, at iteration $t$, when prediction error $e_{t}$ is available, the step-size $\eta_{t}$ can be optimized. This is done by minimizing the instantaneous square error:

$$
\eta_{t}=\eta_{t-1}-\mu \frac{\partial}{\partial \eta_{t-1}}\left[e_{t}^{2}\right]
$$

where $\eta_{t-1}$ is the step-size at iteration $t-1$ and $e_{t}=y_{t}-f_{t-1}\left(\boldsymbol{u}_{t}\right)$,

$$
\begin{aligned}
& \eta_{t}=\eta_{t-1}-\mu \frac{\partial}{\partial \eta_{t-1}}\left[\left(y_{t}-f_{t-1}\left(\boldsymbol{u}_{t}\right)\right)^{2}\right] \\
& \eta_{t}=\eta_{t-1}-\mu \frac{\partial}{\partial \eta_{t-1}}\left[y_{t}^{2}-2 y_{t} f_{t-1}\left(\boldsymbol{u}_{t}\right)+f_{t-1}^{2}\left(\boldsymbol{u}_{t}\right)\right]
\end{aligned}
$$


from Equation (3), we have that $f_{t-1}\left(\boldsymbol{u}_{t}\right)$ can be defined as $f_{t-2}\left(\boldsymbol{u}_{t}\right)+\eta_{t-1} e_{t-1} \kappa_{\sigma}\left(\boldsymbol{u}_{t-1}, \boldsymbol{u}_{t}\right)$,

$$
\begin{aligned}
\eta_{t}= & \eta_{t-1}-\mu \frac{\partial}{\partial \eta_{t-1}}\left[y_{t}^{2}-2 y_{t}\left(f_{t-2}\left(\boldsymbol{u}_{t}\right)+\eta_{t-1} e_{t-1} \kappa_{\sigma}\left(\boldsymbol{u}_{t-1}, \boldsymbol{u}_{t}\right)\right)\right. \\
& \left.+\left(f_{t-2}\left(\boldsymbol{u}_{t}\right)+\eta_{t-1} e_{t-1} \kappa_{\sigma}\left(\boldsymbol{u}_{t-1}, \boldsymbol{u}_{t}\right)\right)^{2}\right] \\
\eta_{t}= & \eta_{t-1}-\mu\left(\frac{\partial}{\partial \eta_{t-1}}\left[y_{t}^{2}\right]-\frac{\partial}{\partial \eta_{t-1}}\left[2 y_{t} f_{t-2}\left(\boldsymbol{u}_{t}\right)\right]\right. \\
& -\frac{\partial}{\partial \eta_{t-1}}\left[2 y_{t} \eta_{t-1} e_{t-1} \kappa_{\sigma}\left(\boldsymbol{u}_{t-1}, \boldsymbol{u}_{t}\right)\right]+\frac{\partial}{\partial \eta_{t-1}}\left[f_{t-2}^{2}\left(\boldsymbol{u}_{t}\right)\right] \\
& +\frac{\partial}{\partial \eta_{t-1}}\left[2 f_{t-2}\left(\boldsymbol{u}_{t}\right) \eta_{t-1} e_{t-1} \kappa_{\sigma}\left(\boldsymbol{u}_{t-1}, \boldsymbol{u}_{t}\right)\right] \\
& \left.+\frac{\partial}{\partial \eta_{t-1}}\left[\left(\eta_{t-1} e_{t-1} \kappa_{\sigma}\left(\boldsymbol{u}_{t-1}, \boldsymbol{u}_{t}\right)\right)^{2}\right]\right)
\end{aligned}
$$

since $f_{t-2}$ does not depend on $\eta_{t-1}$,

$$
\begin{aligned}
& \eta_{t}=\eta_{t-1}-\mu\left(-2\left[y_{t}-f_{t-2}\left(\boldsymbol{u}_{t}\right)-\eta_{t-1} e_{t-1} \kappa_{\sigma}\left(\boldsymbol{u}_{t-1}, \boldsymbol{u}_{t}\right)\right] e_{t-1} \kappa_{\sigma}\left(\boldsymbol{u}_{t-1}, \boldsymbol{u}_{t}\right)\right) \\
& \eta_{t}=\eta_{t-1}-\mu\left(-2\left[y_{t}-\left(f_{t-2}\left(\boldsymbol{u}_{t}\right)+\eta_{t-1} e_{t-1} \kappa_{\sigma}\left(\boldsymbol{u}_{t-1}, \boldsymbol{u}_{t}\right)\right)\right] e_{t-1} \kappa_{\sigma}\left(\boldsymbol{u}_{t-1}, \boldsymbol{u}_{t}\right)\right)
\end{aligned}
$$

note that, $f_{t-2}\left(\boldsymbol{u}_{t}\right)+\eta_{t-1} e_{t-1} \kappa_{\sigma}\left(\boldsymbol{u}_{t-1}, \boldsymbol{u}_{t}\right)$ is actually $f_{t-1}\left(\boldsymbol{u}_{t}\right)$,

$$
\begin{aligned}
\eta_{t} & =\eta_{t-1}-\mu\left(-2\left[y_{t}-f_{t-1}\left(\boldsymbol{u}_{t}\right)\right] e_{t-1} \kappa_{\sigma}\left(\boldsymbol{u}_{t-1}, \boldsymbol{u}_{t}\right)\right) \\
\eta_{t} & =\eta_{t-1}-\mu\left(-2 e_{t} e_{t-1} \kappa_{\sigma}\left(\boldsymbol{u}_{t-1}, \boldsymbol{u}_{t}\right)\right) \\
\eta_{t} & =\eta_{t-1}+2 \mu e_{t} e_{t-1} \kappa_{\sigma}\left(\boldsymbol{u}_{t-1}, \boldsymbol{u}_{t}\right) \\
\eta_{t} & =\eta_{t-1}+\beta e_{t} e_{t-1} \kappa_{\sigma}\left(\boldsymbol{u}_{t-1}, \boldsymbol{u}_{t}\right)
\end{aligned}
$$

Thus, at iteration $t$, the step-size $\eta$ can be calculated using the following sequential update algorithm 8

$$
\eta_{t}=\eta_{t-1}+\beta e_{t} e_{t-1} \kappa_{\sigma}\left(\boldsymbol{u}_{t-1}, \boldsymbol{u}_{t}\right)
$$

Finally, combining Equation (3), Alg. 1, and Equation (5), we propose the

\footnotetext{
${ }^{8}$ Where $\beta=2 \mu$ is a free parameter for the step-size adaptation.
} 
following sequential rule for online prediction?

$$
\left\{\begin{array}{l}
f_{0}=0 \\
e_{t}=y_{t}-f_{t-1}\left(\boldsymbol{u}_{t}\right) \\
f_{t}=f_{t-1}+\eta_{t-1} e_{t} \kappa_{\sigma_{i^{*}, t}}\left(\boldsymbol{u}_{t}, \cdot\right) \\
\eta_{t}=\eta_{t-1}+\beta e_{t} e_{t-1} \kappa_{\sigma_{i^{*}, t}}\left(\boldsymbol{u}_{\left|C_{i^{*}}\right|}, \boldsymbol{u}_{t}\right)
\end{array}\right.
$$

\section{Experimental setting}

We validate the proposed framework for online prediction using mean-squareerror (MSE). The learned filter, at the final iteration, is used to compute the MSE values on each test set, as in [14]. The task is to predict the current value of the sample using the previous ten consecutive samples. For comparison purposes, the proposed framework is contrasted with the following online prediction methods:

1. KLMS [14, which is the simplest and the starting point of many algorithms in the KAF family;

2. KLMS-VSS-1, that uses a variable step-size algorithm [36] in the KLMS method;

3. KLMS-VSS-2, where KLMS is tested with a recently proposed variable step-size strategy [37;

4. NICE [35, which is a recently proposed method that out-performs traditional KAF-based algorithms in the prediction of chaotic time-series;

5. Long short-term memory (LSTM) [6], a type of RNN used to learn sequences of observations. We use the implementation known as vanilla

\footnotetext{
${ }^{9}$ Where $\sigma_{i^{*}, t}$ is the appropriate kernel-size for the sample $\boldsymbol{u}_{t}$ and $\boldsymbol{u}_{\left|C_{i^{*}}\right|}$ is the last sample stored in the dictionary $i^{*}$ (see Alg. 11.
} 
LSTM, as it has shown stable performance when compared with other LSTM variants [38;

6. a regularized RNN proposed in 39 .

\subsection{Data sets}

Testing is carried out on the following three publicly available data sets:

Internet traffic dataset (IT). - This collection holds Internet traffic data from a private service provider with centres in 11 European cities. The data are normalized for the computation convenience, and for implementing the validation strategy, the first 4000 consecutive samples are used as the training set, while another 500 consecutive samples are the test set.

Foreign exchange prices $(F X)$. - This collection has mid-prices (a reference price calculated by taking the average of the current quoted bid and ask prices) for currency pairs EUR/USD and GBP/USD with daily resolution over 12 years. In the simulations: i) the data set is normalized for the computation convenience; ii) the training set covers January 3, 2005, to December 17, 2015, while the test set covers January 4, 2016, to May 18, 2017.

Tesla stock price (TSLA). - This data set shows Tesla's stock price from its initial public offering. The data are normalized for the computation convenience, and predictions are carried out on the stock's closing price. The training set covers June 29, 2010, to June 13, 2016, while the test set covers July 5, 2016, to November 23, 2016.

\subsection{Proposal}

Table 1 summarizes the set-up of our proposal in the tested data sets. To ensure consistency in the results, both step-sizes $\eta$ and $\eta_{1}$ remain the same for all KAF methods. Thus, as long as this condition is met, different step-size values from those shown in Tables 1 and 3 will not offer an advantage to any particular algorithm. The initial kernel-size $\sigma_{1}$ was adjusted using the strategy 
proposed in [14, where the best kernel-size is the one with the lowest MSE value (see Table 2). Note that the same strategy is used to select the best kernel-sizes $\sigma$ in the compared methods (see Section 4.3). The performance of our proposal is sensitive to the selection of $\rho, \beta$, and $\delta$. However, the appropriate values for these parameters can be selected as follows: i) $\rho$-kernel size adaptation, this parameter reflects a trade-off between mis-adjustment and speed of adaptation in Equation (4). An appropriate $\rho$ value, based on our experimentation, is in the interval $[0,1]$; ii) $\beta$-step size adaptation, where a value of 0.0001 has shown stable performance on all tested data sets; iii) $\delta$-centroid threshold, which is a value between 0 and 1 that controls the number of kernel-sizes formed during training. Thus, the greater the value of $\delta$, the more kernel-sizes will be formed.

Table 1: Parameter setting of proposed framework in considered data sets. IT-Internet traffic. $F X$-foreign exchange. TSLA-Tesla stock price. $M$-input vector size, $\eta_{1}$-initial step size, $\sigma_{1}$ initial kernel size, $\rho$-kernel size adaptation, $\beta$-step size adaptation, $\delta$-centroid threshold.

\begin{tabular}{|c|c|c|c|c|c|c|c|}
\hline \multirow{2}{*}{\multicolumn{2}{|c|}{ Dataset }} & \multicolumn{6}{|c|}{ Parameter } \\
\hline & & $M$ & $\eta_{1}$ & $\sigma_{1}$ & $\rho$ & $\beta$ & $\delta$ \\
\hline IT & & 10 & 0.01 & 0.17 & 0.7 & 0.0001 & 0.9 \\
\hline \multirow{2}{*}{ FX } & $E U R / U S D$ & 10 & 0.05 & 0.005 & 0.25 & 0.0001 & 0.95 \\
\hline & $G B P / U S D$ & 10 & 0.05 & 0.35 & 0.35 & 0.0001 & 0.95 \\
\hline \multicolumn{2}{|c|}{ TSLA } & 10 & 0.05 & 0.45 & 0.05 & 0.0001 & 0.05 \\
\hline
\end{tabular}

Table 2: Testing MSE for proposal in considered data sets using different values of initial kernel-sizes $\sigma_{1}$. IT -Internet traffic dataset. FX-foreign exchange. TSLA-Tesla stock price.

\begin{tabular}{|c|c|c|c|c|c|c|}
\hline \multicolumn{2}{|c|}{ IT } & \multicolumn{3}{|c|}{ FX } & \multicolumn{2}{|c|}{ TSLA } \\
\hline$\sigma_{1}$ & MSE & $\sigma_{1}$ & $\begin{array}{c}\text { MSE } \\
(E U R / U S D)\end{array}$ & $\begin{array}{c}\mathrm{MSE} \\
(G B P / U S D)\end{array}$ & $\sigma_{1}$ & MSE \\
\hline 0.005 & 0.0356 & 0.005 & $0.00021 *$ & 0.04530 & 0.005 & 0.00458 \\
\hline 0.17 & $0.0028 *$ & 0.1 & 0.00022 & 0.00046 & 0.1 & 0.00409 \\
\hline 0.28 & 0.0055 & 0.25 & 0.00028 & 0.00047 & 0.25 & 0.00446 \\
\hline 0.39 & 0.0055 & 0.35 & 0.00036 & $0.00032 *$ & 0.35 & 0.00073 \\
\hline 0.445 & 0.0044 & 0.45 & 0.00042 & 0.00041 & 0.45 & $0.00041 *$ \\
\hline 0.5 & 0.0044 & 0.5 & 0.00045 & 0.00055 & 0.5 & 0.00048 \\
\hline
\end{tabular}




\subsection{Reference methods}

The set-up of compared methods is summarized in Table 3 . The parameters $\eta$ and $\eta_{1}$ were adjusted following the analysis described in Section 4.2 . As previously, the kernel-sizes $\sigma$ were selected using the strategy proposed in [14] (see Table 4). The centroid distance $\lambda$ of NICE is set at $2 \sigma$ [35]. The number of neurons $\mathcal{N}$ and epochs $\mathcal{E}$ have been adjusted heuristically using a single hidden layer in LSTM (see Table 5). Note, for better performance, a common NN approach is to add more layers to learn high-level features. However, when depth increases, errors between layers will be accumulated and gradients will vanish, meaning that the network degrades and becomes more difficult to train [40]. Additionally, there is no rule of thumb to select the number of hidden layers in LSTM networks [41, 42, 43]. Thus, here we train the LSTM method using a single hidden layer with the following features: $i$ ) the sigmoid activation function is used for the LSTM blocks; ii) the Adam algorithm [44] is employed for optimization, as suggested in [45]; iii) the mean-square-error is used as a loss function. Lastly, we implement the medium RNN proposed in [39, which has 650 neurons per layer. The NN methods were implemented using TensorFlow (version 1.4.0) ${ }^{10}$ and Keras (version 2.1.2) ${ }^{11}$

\footnotetext{
${ }^{10}$ https://www.tensorflow.org/

${ }^{11}$ https://keras.io/
} 
Table 3: Parameter setting of compared methods in considered data sets. IT-Internet traffic dataset. $F X$-foreign exchange. TSLA-Tesla stock price. $M$-input vector size, $\eta$-step size, $\eta_{1}$ initial step size, $\sigma$-kernel size, $\lambda$-centroid distance, $\mathcal{L}$-layers, $\mathcal{N}$-neurons per layer, $\mathcal{E}$-epochs.

\begin{tabular}{|c|c|c|c|c|c|c|c|c|c|}
\hline \multirow{2}{*}{ Dataset } & \multirow{2}{*}{ Method } & \multicolumn{8}{|c|}{ Parameter } \\
\hline & & $M$ & $\eta$ & $\eta_{1}$ & $\sigma$ & $\lambda$ & $\mathcal{L}$ & $\mathcal{N}$ & $\mathcal{E}$ \\
\hline \multirow{6}{*}{ IT } & KLMS & 10 & 0.01 & - & 0.5 & - & - & - & - \\
\hline & KLMS-VSS-1 & 10 & - & 0.01 & 0.5 & - & - & - & - \\
\hline & KLMS-VSS-2 & 10 & - & 0.01 & 0.5 & - & - & - & - \\
\hline & NICE & 10 & 0.01 & - & 0.39 & $2 \sigma$ & - & - & - \\
\hline & LSTM & 10 & - & - & - & - & 1 & 8 & 50 \\
\hline & RNN & 10 & - & - & - & - & 2 & 650 & 90 \\
\hline \multirow{6}{*}{$E U R / U S D$} & KLMS & 10 & 0.05 & - & 0.1 & - & - & - & - \\
\hline & KLMS-VSS-1 & 10 & - & 0.05 & 0.1 & - & - & - & - \\
\hline & KLMS-VSS-2 & 10 & - & 0.05 & 0.1 & - & - & - & - \\
\hline & NICE & 10 & 0.05 & - & 0.1 & $2 \sigma$ & - & - & - \\
\hline & LSTM & 10 & - & - & - & - & 1 & 8 & 900 \\
\hline & $\mathrm{RNN}$ & 10 & - & - & - & - & 2 & 650 & 200 \\
\hline FX & & & & & & & & & \\
\hline \multirow{6}{*}{$G B P / U S D$} & KLMS & 10 & 0.05 & - & 0.45 & - & - & - & - \\
\hline & KLMS-VSS-1 & 10 & - & 0.05 & 0.45 & - & - & - & - \\
\hline & KLMS-VSS-2 & 10 & - & 0.05 & 0.45 & - & - & - & - \\
\hline & NICE & 10 & 0.05 & - & 0.45 & $2 \sigma$ & - & - & - \\
\hline & LSTM & 10 & - & - & - & - & 1 & 8 & 4000 \\
\hline & $\mathrm{RNN}$ & 10 & - & - & - & - & 2 & 650 & 4000 \\
\hline \multirow{6}{*}{ TSLA } & KLMS & 10 & 0.05 & - & 0.5 & - & - & - & - \\
\hline & KLMS-VSS-1 & 10 & - & 0.05 & 0.5 & - & - & - & - \\
\hline & KLMS-VSS-2 & 10 & - & 0.05 & 0.5 & - & - & - & - \\
\hline & NICE & 10 & 0.05 & - & 0.5 & $2 \sigma$ & - & - & - \\
\hline & LSTM & 10 & - & - & - & - & 1 & 8 & 400 \\
\hline & RNN & 10 & - & - & - & - & 2 & 650 & 400 \\
\hline
\end{tabular}


Table 4: Testing MSE for KAF methods in considered data sets using different kernel-sizes $\sigma$. $I T$-Internet traffic dataset. $F X$-foreign exchange. TSLA-Tesla stock price.

\begin{tabular}{|c|c|c|c|c|c|}
\hline \multirow{2}{*}{ Dataset } & \multirow{2}{*}{$\sigma$} & \multicolumn{4}{|c|}{ Method } \\
\hline & & \multicolumn{2}{|c|}{ KLMS } & \multicolumn{2}{|c|}{ NICE } \\
\hline \multirow{6}{*}{ IT } & 0.005 & \multicolumn{2}{|c|}{0.2630} & \multicolumn{2}{|c|}{0.2630} \\
\hline & 0.17 & \multicolumn{2}{|c|}{0.0116} & \multicolumn{2}{|c|}{0.0178} \\
\hline & 0.28 & \multicolumn{2}{|c|}{0.0055} & \multicolumn{2}{|c|}{0.0062} \\
\hline & 0.39 & \multicolumn{2}{|c|}{0.0044} & \multicolumn{2}{|c|}{0.0050} \\
\hline & 0.445 & \multicolumn{2}{|c|}{0.0040} & \multicolumn{2}{|c|}{0.0048} \\
\hline & 0.5 & \multicolumn{2}{|c|}{$0.0037^{*}$} & \multicolumn{2}{|c|}{$0.0045^{*}$} \\
\hline \multirow{7}{*}{ FX } & & $E U R / U S D$ & $G B P / U S D$ & $E U R / U S D$ & $G B P / U S D$ \\
\hline & 0.005 & 0.01340 & 0.02621 & 0.01340 & 0.02621 \\
\hline & 0.1 & $0.00025 *$ & 0.00433 & $0.00034^{*}$ & 0.00438 \\
\hline & 0.25 & 0.00045 & 0.00192 & 0.00045 & 0.00192 \\
\hline & 0.35 & 0.00045 & 0.00070 & 0.00045 & 0.00069 \\
\hline & 0.45 & 0.00046 & $0.00035^{*}$ & 0.00045 & $0.00035^{*}$ \\
\hline & 0.5 & 0.00048 & 0.00062 & 0.00048 & 0.00062 \\
\hline \multirow{6}{*}{ TSLA } & 0.005 & \multicolumn{2}{|c|}{0.49561} & \multicolumn{2}{|c|}{0.49561} \\
\hline & 0.1 & \multicolumn{2}{|c|}{0.00937} & \multicolumn{2}{|c|}{0.02213} \\
\hline & 0.25 & \multicolumn{2}{|c|}{0.00170} & \multicolumn{2}{|c|}{0.00181} \\
\hline & 0.35 & \multicolumn{2}{|c|}{0.00082} & \multicolumn{2}{|c|}{0.00131} \\
\hline & 0.45 & \multicolumn{2}{|c|}{0.00045} & \multicolumn{2}{|c|}{0.00043} \\
\hline & 0.5 & \multicolumn{2}{|c|}{$0.00045 *$} & \multicolumn{2}{|c|}{$0.00042^{*}$} \\
\hline
\end{tabular}


Table 5: Testing MSE for NN methods in considered data sets using different epochs $\mathcal{E}$. $I T$-Internet traffic dataset. $F X$-foreign exchange prices. TSLA-Tesla stock price. $\mathcal{N}$-neurons.

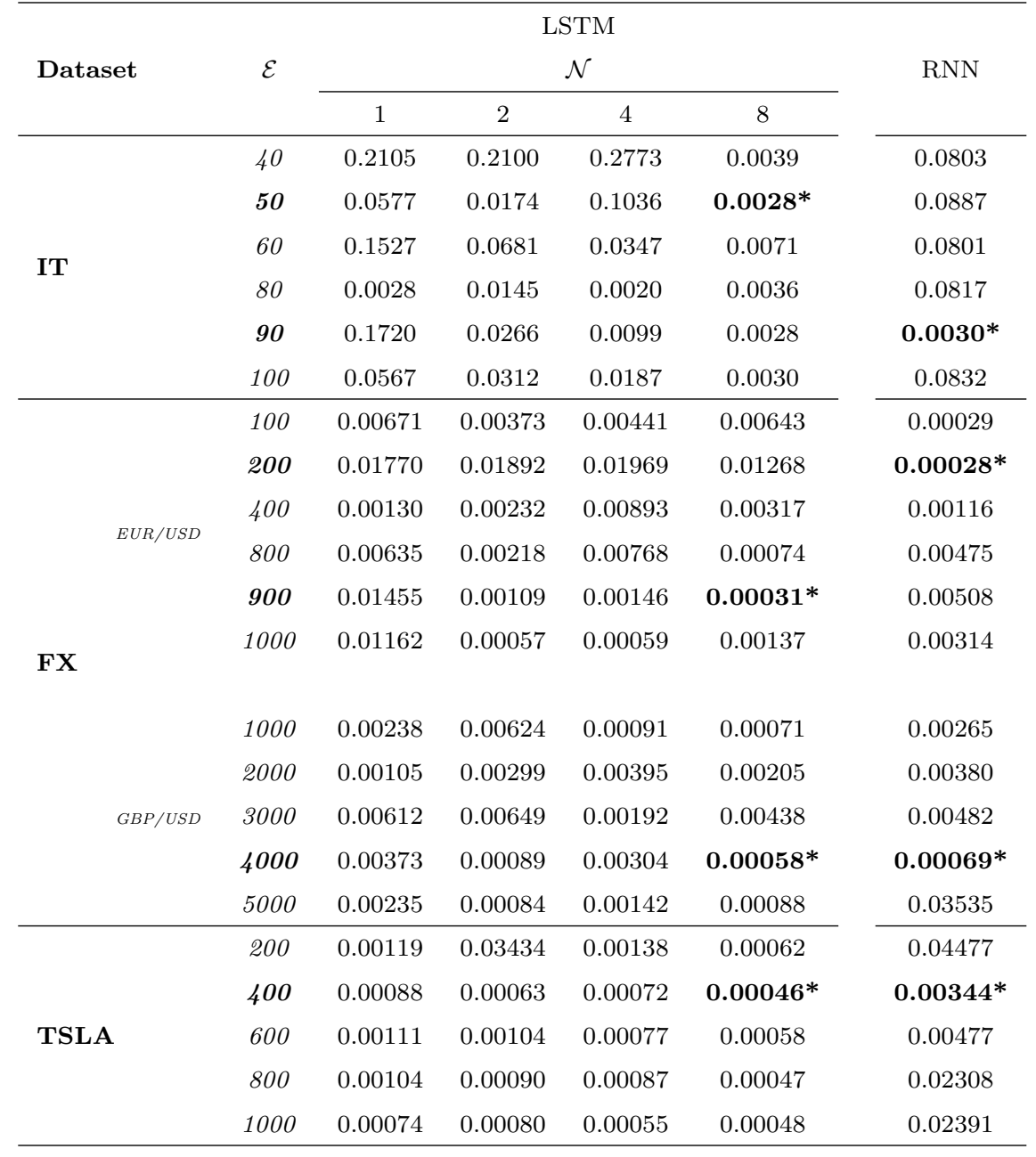




\section{Results}

We present quantitative and visual results of the proposed framework for online prediction along with the reference methods. The results give performance of the corresponding model on each test set (see Section 4.1).

Table 6 summarizes the results found in Sections 4.2 and 4.3. We see that our proposal outperforms the other algorithms on all considered data sets, converging to smaller values of MSE, which suggests that the proposed adaptive step-size helps to improve convergence time. Of note, even though LSTM and our proposal achieve similar MSE values on Internet traffic prediction (IT), LSTM requires a significantly higher number of samples to enable predictions in the test set. Additionally, NN models must be retrained regularly in sequence prediction tasks, which requires both significant computing and storage resources [13]. That is, both algorithms (LSTM and RNN) need to pass the entire training set both forward and backward through the $\mathrm{NN}$ - also known as an epoch. This process is usually repeated several times during the learning stage. Thus, in real-time prediction tasks, the training set will be updated with

every new sample that arrives in the system, meaning that several epochs have to be re-performed to find the best possible performance each time the training set is updated. The learning scheme of KAF methods, unlike LSTM and RNN, is a combination of error-correction and memory-based learning, which means that they do not require the whole training set to start learning the model. In contrast, as the samples arrive, predictions are obtained while the model is updated sequentially at the same time, which is useful in real-time applications. Thus, although the concept of epoch is not used in KAF methods [14, this can be seen as an attempt to learn the network topology in a single epoch, i.e., the model is learned using a single pass through the entire training set in KAF algorithms. 
Table 6: Testing MSE in considered data sets. MSE-mean squared error. Samples-average number of samples used to predict test set. IT-Internet traffic dataset. $F X$-foreign exchange prices. TSLA-Tesla stock price. The bold notation indicates the best method.

\begin{tabular}{|c|c|c|c|c|c|}
\hline Dataset & Method & \multicolumn{2}{|c|}{ Testing MSE } & \multicolumn{2}{|c|}{ Samples } \\
\hline & KLMS & \multicolumn{2}{|c|}{0.0037} & \multicolumn{2}{|c|}{$4000 \pm 0$} \\
\hline & KLMS-VSS-1 & \multicolumn{2}{|c|}{0.0034} & \multicolumn{2}{|c|}{$4000 \pm 0$} \\
\hline & KLMS-VSS-2 & \multicolumn{2}{|c|}{0.0044} & \multicolumn{2}{|c|}{$4000 \pm 0$} \\
\hline IT & NICE & \multicolumn{2}{|c|}{0.0045} & \multicolumn{2}{|c|}{$2302.7 \pm 821.8$} \\
\hline & LSTM & \multicolumn{2}{|c|}{0.0028} & \multicolumn{2}{|c|}{$4000 \pm 0$} \\
\hline & RNN & \multicolumn{2}{|c|}{0.0030} & \multicolumn{2}{|c|}{$4000 \pm 0$} \\
\hline & Proposal & \multicolumn{2}{|c|}{0.0028} & \multicolumn{2}{|c|}{$920.4 \pm 462.6$} \\
\hline \multirow{8}{*}{ FX } & & $E U R / U S D$ & $G B P / U S D$ & $E U R / U S D$ & $G B P / U S D$ \\
\hline & KLMS & 0.00025 & 0.00035 & $4000 \pm 0$ & $4000 \pm 0$ \\
\hline & KLMS-VSS-1 & 0.00056 & 0.00036 & $4000 \pm 0$ & $4000 \pm 0$ \\
\hline & KLMS-VSS-2 & 0.00355 & 0.00050 & $4000 \pm 0$ & $4000 \pm 0$ \\
\hline & NICE & 0.00034 & 0.00035 & $607.9 \pm 6.5$ & $4000 \pm 0$ \\
\hline & LSTM & 0.00031 & 0.00058 & $4000 \pm 0$ & $4000 \pm 0$ \\
\hline & RNN & 0.00028 & 0.00069 & $4000 \pm 0$ & $4000 \pm 0$ \\
\hline & Proposal & 0.00021 & 0.00032 & $347.2 \pm 25.2$ & $440.3 \pm 5.4$ \\
\hline \multirow{7}{*}{ TSLA } & KLMS & \multicolumn{2}{|c|}{0.00045} & \multicolumn{2}{|c|}{$1500 \pm 0$} \\
\hline & KLMS-VSS-1 & \multicolumn{2}{|c|}{0.00223} & \multicolumn{2}{|c|}{$1500 \pm 0$} \\
\hline & KLMS-VSS-2 & \multicolumn{2}{|c|}{0.00652} & \multicolumn{2}{|c|}{$1500 \pm 0$} \\
\hline & $\mathrm{NICE}$ & \multicolumn{2}{|c|}{0.00042} & \multicolumn{2}{|c|}{$1363.3 \pm 216.7$} \\
\hline & LSTM & \multicolumn{2}{|c|}{0.00046} & \multicolumn{2}{|c|}{$1500 \pm 0$} \\
\hline & RNN & \multicolumn{2}{|c|}{0.00343} & \multicolumn{2}{|c|}{$1500 \pm 0$} \\
\hline & Proposal & \multicolumn{2}{|c|}{0.00041} & \multicolumn{2}{|c|}{$1369 \pm 214$} \\
\hline
\end{tabular}

The column "Samples" in Table 6 gives the average number of samples used to predict the test sets. These samples come from the learned filter at the final iteration. It is clear that KLMS, KLMS-VSS-1, KLMS-VSS-2, LSTM, and $\mathrm{RNN}$ use all the samples from the training sets to derive predictions on the test sets. These algorithms do not have a sparsification technique, which is a major drawback. For example, from the point of view of KLMS, the current dynamic of the system does not matter. Consequently, each sample in the test set of the FX dataset is predicted using all 4000 samples that were learned during training. This results in high computational complexity in online applications, i.e., the KLMS dictionary grows linearly with each new sample. In contrast, our proposal uses a moderate number of samples to obtain each prediction in the test 
set, suggesting that the proposed multiple kernel-sizes strategy retains the most relevant samples to perform later prediction tasks effectively. Additionally, the knowledge-transfer strategy of our framework, compared to that used by the NICE algorithm, reduces the required number of samples without significant loss of accuracy (see Table 6).

Figure 3 shows the predicted signals in the test sets. Figures $3(\mathrm{a})$ and $3(\mathrm{~d})$ display the predictions on the Internet traffic dataset and Tesla stock prices, respectively, while Figures 3(b) and 3(c) show the results for FX prices. The multiple kernel-sizes strategy and the adaptive step-size, incorporated in our framework, enable the kernel-based adaptive filter to converge more quickly while competitive performance is maintained on all tested data sets. However, if the initial kernel-size $\sigma_{1}$ is inappropriately chosen, the prediction accuracy may be adversely affected (see Table 2). In this case, the suitable initial value of $\sigma_{1}$ can be selected using a method such as Silverman's rule [14]. The predictions of our proposal, as seen in Figure 3 , are very close to the desired signals. Therefore, the proposed framework shows the lowest MSE values in Table 6, proving its stable performance in real-world applications. 


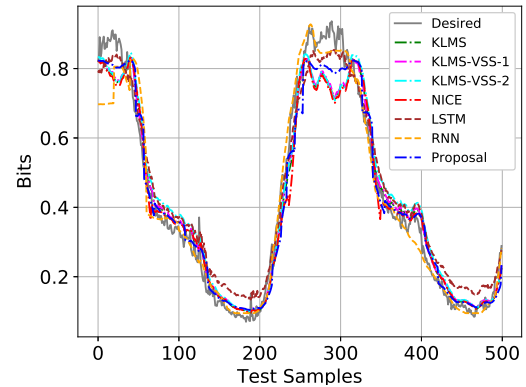

(a) Internet traffic

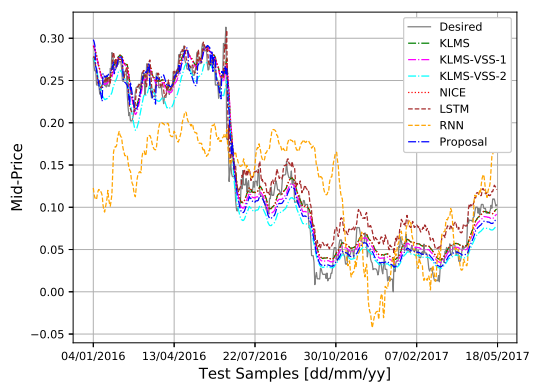

(c) GBP/USD

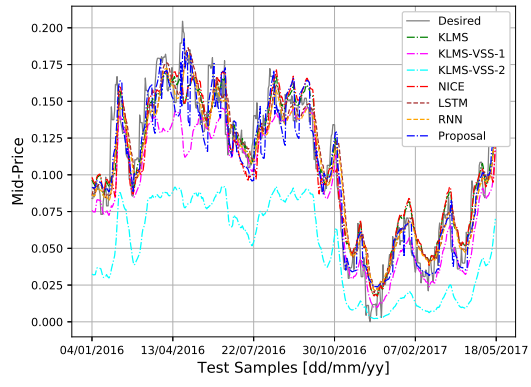

(b) EUR/USD

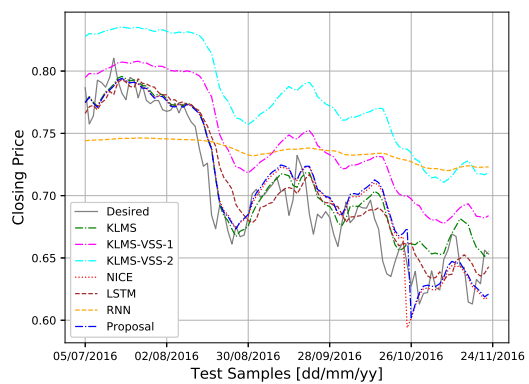

(d) Tesla stock price

Figure 3: Performed predictions in the considered data sets.

\section{Conclusions}

This study introduces a framework for online prediction that addresses two of the main challenges of KAFs: selection of appropriate kernel-size and stepsize. The proposed multiple kernel-sizes and the adaptive step-size strategies are combined, improving convergence time while competitive performance is maintained. However, we must clarify that our framework may be adversely affected in the case of relatively few training samples, as it is more difficult to identify hidden patterns from data under this scenario. The minimum number of samples, based on our experiments, required to train highly non-stationary signals is 1500 .

This work sequentially updates the kernel-size and step-size parameters us- 
ing stochastic gradient algorithms that minimize MSE. The kernel-size problem is addressed from a practical application focus; whilst the strategy may seem relatively simple, to the best of our knowledge, it has not been previously proposed. Further, the results show a general improvement in prediction accuracy in online sequential learning environments.

The proposed framework can be incorporated into any KLMS-based algorithm. To test its performance we have used three real-world data sets. Simulations results demonstrate that our proposal learns from a continuous sequence of data records, adapts to changing statistics in the data, exhibits high tolerance to noisy conditions and provides stable performance in real-world applications.

We are in the process of expanding our research to the case where several Mercer kernels (not restricted to the Gaussian kernel) are combined together to support online prediction tasks. 


\section{Acknowledgement}

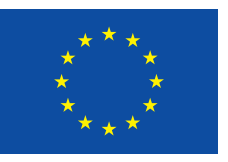

This project has received funding from the European Union's

Horizon 2020 research and innovation programme under the Marie Skodowska-Curie grant agreement No 675044.

\section{References}

[1] T. Gueniche, P. Fournier-Viger, R. Raman, V. S. Tseng, Cpt+: Decreasing the time/space complexity of the compact prediction tree, in: Pacific-Asia Conference on Knowledge Discovery and Data Mining, Springer, 2015, pp. 625-636.

[2] A. Jobava, A. Yazidi, B. J. Oommen, K. Begnum, On achieving intelligent traffic-aware consolidation of virtual machines in a data center using learning automata, Journal of Computational Science 24 (2018) 290 - 312.

[3] S. M. Vahidipour, M. Esnaashari, Priority assignment in queuing systems with unknown characteristics using learning automata and adaptive stochastic petri nets, Journal of Computational Science 24 (2018) 343 357.

[4] M. Dixon, Sequence classification of the limit order book using recurrent neural networks, Journal of Computational Science 24 (2018) 277 - 286.

[5] S. Ahmad, A. Lavin, S. Purdy, Z. Agha, Unsupervised real-time anomaly detection for streaming data, Neurocomputing 262 (2017) 134-147.

[6] S. Hochreiter, J. Schmidhuber, Long short-term memory, Neural computation 9 (8) (1997) 1735-1780.

[7] E. Tsironi, P. Barros, C. Weber, S. Wermter, An analysis of convolutional long short-term memory recurrent neural networks for gesture recognition, Neurocomputing 268 (2017) 76-86. 
[8] M. A. Mohammed, M. K. A. Ghani, R. I. Hamed, D. A. Ibrahim, M. K. Abdullah, Artificial neural networks for automatic segmentation and identification of nasopharyngeal carcinoma, Journal of Computational Science 21 (2017) $263-274$.

[9] J. H. Tan, U. R. Acharya, S. V. Bhandary, K. C. Chua, S. Sivaprasad, Segmentation of optic disc, fovea and retinal vasculature using a single convolutional neural network, Journal of Computational Science 20 (2017) $70-79$.

[10] A. A. Ahmed, M. F. Mohammed, Sairf: A similarity approach for attack intention recognition using fuzzy min-max neural network, Journal of Computational Science 25 (2018) $467-473$.

[11] K. Bouallegue, A new class of neural networks and its applications, Neurocomputing 249 (2017) 28-47.

[12] S. B. Taieb, G. Bontempi, A. F. Atiya, A. Sorjamaa, A review and comparison of strategies for multi-step ahead time series forecasting based on the nn5 forecasting competition, Expert systems with applications 39 (8) (2012) 7067-7083.

[13] Y. Cui, S. Ahmad, J. Hawkins, Continuous online sequence learning with an unsupervised neural network model, Neural computation 28 (11) (2016) $2474-2504$.

[14] W. Liu, J. C. Principe, S. Haykin, Kernel Adaptive Filtering: A Comprehensive Introduction, 1st Edition, Wiley Publishing, 2010.

[15] J. C. Principe, Information theoretic learning: Renyi's entropy and kernel perspectives, Springer Science \& Business Media, 2010.

[16] F. Orabona, J. Keshet, B. Caputo, Bounded kernel-based online learning, Journal of Machine Learning Research 10 (Nov) (2009) 2643-2666. 
[17] P. Zhao, S. C. Hoi, R. Jin, Double updating online learning, Journal of Machine Learning Research 12 (May) (2011) 1587-1615.

[18] J. Tang, J. Zhang, Z. Wu, Z. Liu, T. Chai, W. Yu, Modeling collinear data using double-layer ga-based selective ensemble kernel partial least squares algorithm, Neurocomputing 219 (2017) 248-262.

[19] W. Liu, P. P. Pokharel, J. C. Principe, The kernel least-mean-square algorithm, IEEE Transactions on Signal Processing 56 (2) (2008) 543-554.

[20] W. Liu, J. C. Príncipe, Kernel affine projection algorithms, EURASIP Journal on Advances in Signal Processing 2008 (1) (2008) 784292.

[21] B. Chen, S. Zhao, P. Zhu, J. C. Príncipe, Quantized kernel least mean square algorithm, IEEE Transactions on Neural Networks and Learning Systems 23 (1) (2012) 22-32.

[22] K. Li, J. C. Príncipe, The kernel adaptive autoregressive-moving-average algorithm, IEEE transactions on neural networks and learning systems 27 (2) (2016) 334-346.

[23] B. Chen, J. Liang, N. Zheng, J. C. Principe, Kernel least mean square with adaptive kernel size, Neurocomputing 191 (2016) 95-106.

[24] S. An, W. Liu, S. Venkatesh, Fast cross-validation algorithms for least squares support vector machine and kernel ridge regression, Pattern Recognition 40 (8) (2007) 2154 - 2162, part Special Issue on Visual Information Processing.

[25] W. Härdle, Applied nonparametric regression, no. 19, Cambridge university press, 1990.

[26] E. Herrmann, Local bandwidth choice in kernel regression estimation, Journal of Computational and Graphical Statistics 6 (1) (1997) 35-54.

[27] B. W. Silverman, Density estimation for statistics and data analysis, Vol. 26, CRC press, 1986. 
[28] R. Pokharel, S. Seth, J. C. Principe, Mixture kernel least mean square, in: Neural Networks (IJCNN), The 2013 International Joint Conference on, IEEE, 2013, pp. 1-7.

[29] S. Zhao, B. Chen, P. Zhu, J. C. Príncipe, Fixed budget quantized kernel least-mean-square algorithm, Signal Processing 93 (9) (2013) 2759-2770.

[30] Y. Zheng, S. Wang, J. Feng, K. T. Chi, A modified quantized kernel least mean square algorithm for prediction of chaotic time series, Digital Signal Processing 48 (2016) 130-136.

[31] B. Scholkopf, A. J. Smola, Learning with Kernels: Support Vector Machines, Regularization, Optimization, and Beyond, MIT Press, Cambridge, MA, USA, 2001.

[32] C. Saide, R. Lengelle, P. Honeine, C. Richard, R. Achkar, Nonlinear adaptive filtering using kernel-based algorithms with dictionary adaptation, International Journal of Adaptive Control and Signal Processing 29 (11) (2015) 1391-1410.

[33] C. Richard, J. C. M. Bermudez, P. Honeine, Online prediction of time series data with kernels, IEEE Transactions on Signal Processing 57 (3) (2009) 1058-1067.

[34] W. Gao, J. Chen, C. Richard, J. Huang, Online dictionary learning for kernel lms, IEEE Transactions on Signal Processing 62 (11) (2014) 27652777 .

[35] K. Li, J. C. Príncipe, Transfer learning in adaptive filters: The nearest instance centroid-estimation kernel least-mean-square algorithm, IEEE Transactions on Signal Processing 65 (24) (2017) 6520-6535.

[36] Y. Li, M. Hamamura, Zero-attracting variable-step-size least mean square algorithms for adaptive sparse channel estimation, International Journal of Adaptive Control and Signal Processing 29 (9) (2015) 1189-1206. 
[37] Q. Niu, T. Chen, A new variable step size lms adaptive algorithm, in: 2018 Chinese Control And Decision Conference (CCDC), IEEE, 2018.

[38] K. Greff, R. K. Srivastava, J. Koutník, B. R. Steunebrink, J. Schmidhuber, Lstm: A search space odyssey, IEEE transactions on neural networks and learning systems 28 (10) (2017) 2222-2232.

[39] W. Zaremba, I. Sutskever, O. Vinyals, Recurrent neural network regularization, in: International Conference on Learning Representations (ICLR), 2015.

[40] J. Wang, B. Peng, X. Zhang, Using a stacked residual lstm model for sentiment intensity prediction, Neurocomputing 322 (2018) 93-101.

[41] W. Bao, J. Yue, Y. Rao, A deep learning framework for financial time series using stacked autoencoders and long-short term memory, PloS one 12 (7) (2017) e0180944.

[42] H. Palangi, L. Deng, Y. Shen, J. Gao, X. He, J. Chen, X. Song, R. Ward, Deep sentence embedding using long short-term memory networks: Analysis and application to information retrieval, IEEE/ACM Transactions on Audio, Speech and Language Processing (TASLP) 24 (4) (2016) 694-707.

[43] H. Palangi, R. K. Ward, L. Deng, Distributed compressive sensing: A deep learning approach., IEEE Trans. Signal Processing 64 (17) (2016) 45044518.

[44] D. P. Kingma, J. Ba, Adam: A method for stochastic optimization, arXiv preprint arXiv:1412.6980.

[45] Y. Tian, K. Zhang, J. Li, X. Lin, B. Yang, Lstm-based traffic flow prediction with missing data, Neurocomputing 318 (2018) 297-305. 